\title{
Visualization of Virtual Plants Growth Based on Open L-System
}

\author{
Yingying Liu, Juan Pan, Li Yang, Xiaodong Zhu, and Na Zhang* \\ Department of Computer and Information Engineering, \\ Beijing University of Agriculture, Beijing, P.R. China 102206 \\ liuyingying@bac.edu.cn
}

\begin{abstract}
Based on the improvement of Open L-system, this paper presents a four-layer model of plant growth visualization. Take Radix Isatidis for example, the paper carries on morphologic model of its base leaves growing period and eco-physiological model about temperature and growth rate. The prototype system design and development are also discussed on the base of this four-layer model. The results show that the four-layer model solves the combination of the plant morphologic model and eco-physiological model, with some reference value and practical significance.
\end{abstract}

Keywords: virtual plant, Open L-system, modeling, visualization.

\section{Introduction}

Virtual plants center on the research of plant individual, establish three-dimensional model with a focus on the research of plant morphology and visually reflect the physiological and ecological characteristics of crops. [1].

Many scholars at home and abroad have done a great deal researches about morphologic and eco-physiological modeling of virtual plants and have made great progress. But there is still an urgent need for researchers to solve many problems. One of the key issues is to systematically and organically integrate morphologic model and eco-physiological model into growth model. [2] Based on the improvement of Open L-system, this paper puts forward the four-layer model and focuses on solving the combination of the plant morphologic model and eco-physiological model.

\section{Analysis and Improvement of Open L-System}

\subsection{About Open L-System}

Lindenmayer system [3] was designed by Aristid Lindenmayer in 1968 to simulate biological forms, named after himself, and was called L-system for short. L-system is more suitable for the description of fractal phenomenon and also suitable for the

\footnotetext{
Corresponding author.
} 
description of plant morphology. Based on the original L-system (determinacy OLsystem, random L-system, parameter L-system and so on), Open L-system builds an interactive information mode between plants and external conditions by introducing the function expression $\mathrm{E}(\mathrm{x} 1, \mathrm{x} 2, \ldots, \mathrm{xn})$.

Open L-system is usually regarded as an ordered six-tuple shown in formula (1) as followed [4].

$$
\mathrm{G}=\left(\mathrm{V}, \Sigma, \prod, \quad \mathrm{E}, \omega, \mathrm{P}\right) .
$$

In which, $\mathrm{V}$ is character set of system; $\Sigma$ is formal parameter set; $\Pi$ is random probability effect function set, it matches the probability that each production is applied, the sum of each function value is one; $\mathrm{E}$ is the information transfer function to transfer information between plants and condition; $\omega$ is called axiom component of non-empty character; $\mathrm{P}$ is a finite production set.

\subsection{Defect Analysis}

After analyzing how the open L-system works, the author thinks that the open Lsystem has some insufficient s summed up as follows [5].

Inherited Defect. Open L-system inherits the fundamental features of L-system like other L-systems, so it is necessary for users to know well character string rewrite rules and have the skill of converting the rules of plant development into complex productions. Representing geometric construction as well as topological structure of plants in this formal language, especially describing the extremely complicated process of growth makes the production more difficult to comprehend, and it is a trouble for the researchers.

Extensional Defect. Open L-system integrates the processing information such as calculating functions for interacting with external environment, so the indigestible Lsystem becomes more complicated. From the perspective of software engineering it reduces the expandability of the software to a great degree, and it doesn't correspond with modularization principle. It's a taboo that the whole program need modify when processing functions change or development parameters vary.

\subsection{Improvement Research}

Based on the analysis above, the improvement research is focused on "slimming" that mainly reflects in the following two aspects.

Optimizing and Simplifying the Character Set. It is the first step to optimize V in formula (1). When the research object is one specific plant it is reasonable to keep necessary expression symbols and cut down those unnecessary according the growth 
rules. For example, for the visualization of Radix Isatidis base leaf growth, it's a good idea to keep those basic symbols such as rotation angle, increase and decrease angle, beginning of one leaf, end of one leaf, drawing a leaf and returning to origin.

Splitting the Interactive Processing Units off. The next modification is to slip the E and $\prod$ in formula (1) off the open L-system and put them into another modular. So the open L-system is transformed as formula (2).

$$
\mathrm{G}=(\mathrm{V}, \quad \Sigma, \omega, \mathrm{P})
$$

The formal parameters in $\Sigma$ are interfaces between morphogenetic model and ecophysiological model. Eco-physiological model processes growth parameters in give condition according to some growth rules even agricultural expert system. Then those parameters are transferred to open L-system as new plants morphological parameters, so L-system can rewrite character string according to new parameters to represent new state that plants interact with environment.

Some advantages can be manifested by handling in this way.

1. The complexity of open L-system is lower.

2. The degree of coupling between morphogenesis model and eco-physiological model is reduced and the expandability of software is increased.

3. Visualization modular and interactive modular can be parallel processed, so the performance of the system can be improved.

\section{Four-Layer Model Architecture}

Followed the research results above, one integrated framework for virtual plant growth visualization system is presented with the purpose of combination of morphogenetic model and eco-physiological model as shown in fig.1. In this framework, one middle layer is introduced besides the eco-physiological layer, morphological layer and visualization layer. Like OSI/RM or TCP/IP network architecture, layers in the framework coordinate each other in C/S way.

\subsection{Eco-physiological Layer}

Eco-physiological layer mainly imitates the interaction between plants and environments, such as the effect of temperature, humidity or photometric quantity on plant growth and it can also deal with the competition mechanism of plant themselves and feedback to environment from plant. This layer receives the data processed by the middle layer or feedback from plant, and then through plant growth mechanism model even expert system it computes the specific parameters for plant growth. The parameters are transferred to the middle layer later, and then should be transferred to morphological layer, so the interaction between plant and environment can be obtained. 


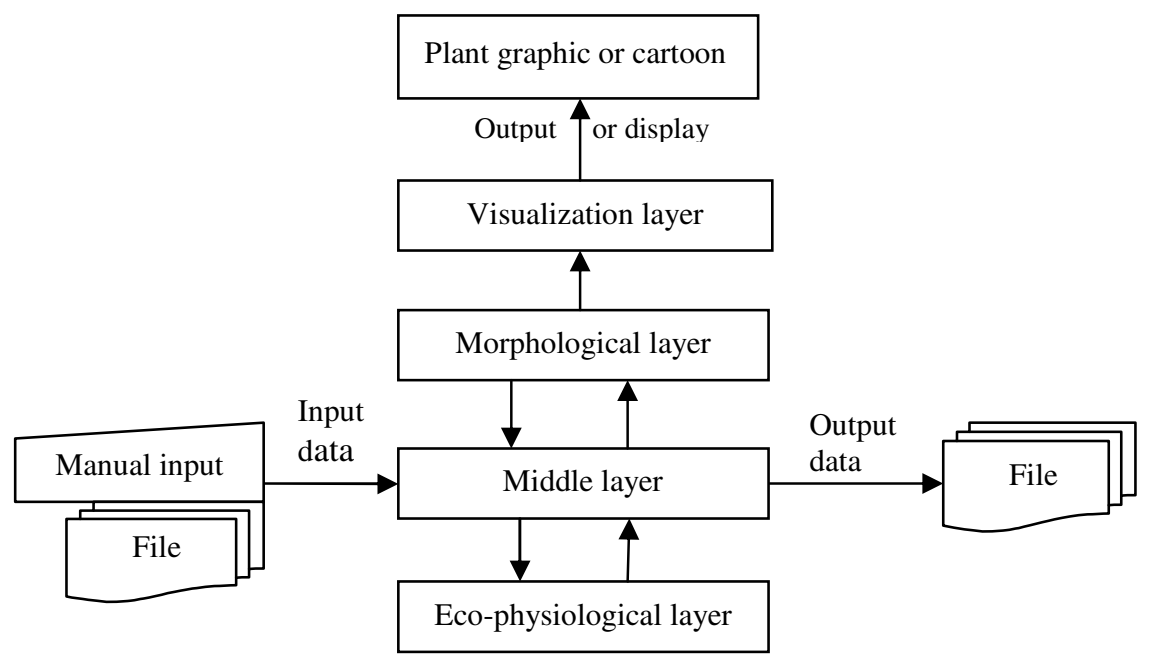

Fig. 1. Four-layer model architecture for virtual plant growth visualization system

\subsection{Morphogenetic Layer}

Based on open L-system, the morphogenetic layer represents the topological and geometric structure by character strings and productions. The interface of plant and environment is held as parameters in the open L-system improved and the middle layer will transfer the parameters processed by the eco-physiological layer through the interface, so eco-physiological model and morphogenetic model integrate well.

\subsection{Visualization Layer}

On the basic of the data processed by morphogenetic layer the visualization layer takes advantage of computer graphics techniques such as geometrical processing, lighting, texture and rendering to show the plant growth in three-dimensional visualization way.

\subsection{Middle Layer}

The middle layer plays a certain role in the nexus. It transfers the definite parameters between morphogenetic layer and eco-physiological layer. Meanwhile, the middle layer is charged with an important mission to receive extern data or output and store data in file or database which is convenient for statistic and analysis later.

\section{$4 \quad$ Implementation and Results}

Design and Implementation of one proto software are discussed in the following section. Radix Isatidis is taken as research object in this software which validates the 
feasibility of four-layer model. The focus is only on the base leaf growth stage of Radix Isatidis in consideration of operability.

\subsection{Design of Eco-physiological Layer}

It is "accumulated temperature method" that was adopted to map the leaf count and temperature .For one single leaf, normality model and "clock model" were introduced to define the function of growth rate and temperature as shown in formula (3).

$$
V(\mathrm{D})=\left(\frac{T-T \min }{T o-T \min }\right)^{-2}\left(\frac{T \max -T}{T \max -T o}\right)^{-2\left(\frac{T \max -T 0}{T o-T \min }\right)} \frac{1}{11 \sqrt{2 \pi}} \operatorname{EXP}\left(-\frac{(\mathrm{D}-11)^{2}}{2 \times 6.65^{2}}\right)
$$

In which $\mathrm{T}=$ average temperature of one day; $\mathrm{Tmin}=0$, the $\min$ temperature that base leaf can live; $T \max =35$, the $\max$ temperature that base leaf can live; $\mathrm{To}=17$, the temperature best fit base leaf; $\mathrm{P}=-2$, impact factor; $\mathrm{D}=$ days.

Furthermore, the functions between growth rate and growth parameters such as length, width, stretch, crimp and color are also defined. Due to space limitations, these elements do not go into details.

\subsection{Process of Morphogenetic Layer}

Based on NURBS the whole base leaf model is built, and the specific leaf appearance was played by a $7 \times 7 \times 3$ parameterized array shown as formula(4), formula(5) and formula(6). This method ensures the blade fluency and clear biological significance.

$$
\begin{gathered}
c[i][j][0]=(j-3) \frac{i w}{6} \\
c[i][j][1]=\frac{i w}{2} \tan \left(\frac{1}{3}\left(\frac{\pi}{2}-\frac{A C \pi}{360}\right) A B S(j-3)\right) \\
c[i][j][1]=(\mathrm{L}-\text { Lstalk }) \frac{i}{6}
\end{gathered}
$$

In addition, basic characters and productions were also defined. As the space is limited, relevant information is leaved out.

\subsection{Design of Middle Layer}

First, both file and database as I/O manner are introduced in this software. The data in database is stored in table, and each table is correlative. The conceptual structure of database is shown as table1.

On the other hand, parallel processing and multithreading technologies are adopted in the software to display morphology results and compute physiological results in parallel. Then parameters are processed into a suitable form, and then transferred between morphology layer and eco-physiological layer. 
Table 1. The conceptual structure of database

\begin{tabular}{lll}
\hline Name of relation & Attributes & Key \\
\hline day & dayid,min,max,s,phaseid & dayid \\
phase & phaseid,s & phaseid \\
topology & dayid,baseleaf,stemleaf,branch,flower,fruit & dayid \\
baseleaf & dayid,leafid,days,len,wid,as,ac,r,g,b & dayid,leafid \\
\hline
\end{tabular}

\subsection{Visualization Technology}

Based on VC++ development platform, twenty-seven classes are defined and implemented. One single Radix Isatidis is drawn according to the hierarchical model based on matrix stack and transformation of open GL. Relevant functions about illumination of open GL are adopted to achieve the illumination and color change.

The prototype system interface is similar to VC style shown as fig.2. Besides the menu and tool bar above, left part is control area concluding three switchable areas that display the parameters of static status display, dynamic display and group display and the right part is graphic display area.

\section{$5 \quad$ Conclusion and Discussion}

The prototype system shows that the four-layer model can well solve the combination of morphology model and eco-physiological model. The four-layer model that encapsulates the eco-physiological model in another layer instead of open L-system, so it is coupling efficiency, scalable and easy to be modified and restructured. In the background of the agriculture and forestry application software, it has certain reference value and practical value.

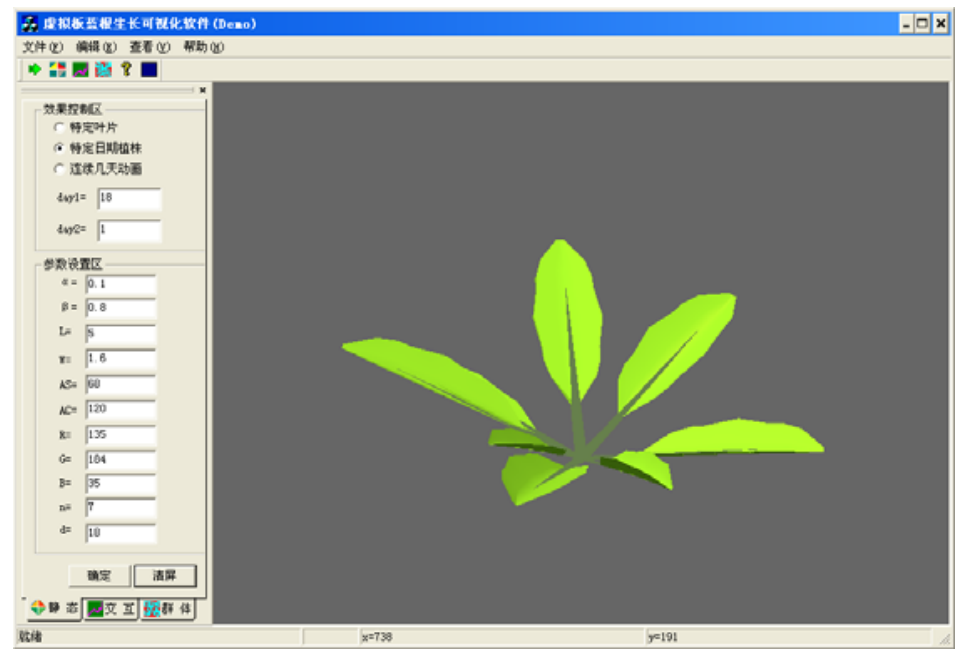

Fig. 2. Interface of the proto software 
This paper is just a try and exploration in a smaller range and further research work is required. Based on perfecting morphology model and eco-physiological model, phototropism of plants, collision detection and realistic graphics generation need to be considered in the following research. In addition, agricultural experts system can consider to be combined, so they will complement each other and guide agricultural production in a better way.

\section{References}

1. Zhou, S.Q.: Virtual plants modeling and visualization. Electronic Industry Press, Beijing (2008) (in Chinese)

2. Hu, B.G., Zhao, X., Yan, H.P., de Reffye, P., Blaise, F., Xiong, F.L., Wang, Y.M.: Plant development model and visualization-review and prospect. Acta 27(6), 816-835 (2001) (in Chinese)

3. Room, P., Hanan, J., Prusinkiewicz, P.: Virtual plants: new perspectives for ecologists, pathologists and agricultural scientists. Trends in Plant Science 1(1), 33-38 (1996)

4. Prusinkiewicz, P.: Modeling plant growth and development. Current Opinion in Plant Biology 7, 79-83 (2004)

5. Liu, Y.Y.: Visualization of Virtual Plants Development Based on Open L System. North China Electric Power University, Beijing (2009) (in Chinese) 